\title{
Optimal Sizing and Control of Energy Storage in Wind Power-Rich Distribution Networks
}

\author{
Sahban W. Alnaser, Graduate Student Member, IEEE, and Luis F. Ochoa, Senior Member, IEEE
}

\begin{abstract}
This paper presents a planning framework to find the minimum storage sizes (power and energy) at multiple locations in distribution networks to reduce curtailment from renewable distributed generation (DG), specifically wind farms, whilst managing congestion and voltages. A two-stage iterative process is adopted in this framework. The first stage uses a multiperiod AC Optimal Power Flow (OPF) across the studied horizon to obtain initial storage sizes considering hourly wind and load profiles. The second stage adopts a high granularity minute-byminute control driven by a mono-period bi-level AC OPF to tune the first-stage storage sizes according to the actual curtailment. Congestion and voltages are managed through the optimal control of storage (active and reactive power), on-load tap changers (OLTCs), DG power factor, and DG curtailment as last resort. The proposed storage planning framework is applied to a real $33 \mathrm{kV}$ network from the North West of England over one week. The results highlight that by embedding high granularity control aspects into planning it is possible to more accurately size storage facilities. Moreover, intelligent management of further flexibility (i.e., OLTCs, storage and DG power factor control) can lead to much smaller storage capacities. This, however, depends on the required level of curtailment.

Index Terms-- Active network management, distributed generation, energy storage, generation curtailment, optimal power flow, wind power.
\end{abstract}

\section{INTRODUCTION}

$\mathrm{A}$ CTIVE Network Management (ANM) has the potential to facilitate the integration of large volumes of renewable Distributed Generation (DG) without the need of traditional reinforcements [1]. However, generation curtailment might be needed to manage congestion and voltage issues. This means that when high penetrations of DG capacity exist within the same distribution network, some DG plants could be subject to significant levels of curtailment that, in turn, may compromise their profitability. Hence, to ensure adequate levels of curtailment, advanced technical solutions are required [2-3].

In this context, battery energy storage is considered to be a key technology in the near future. Indeed, network issues could be actively managed in real-time by storing the excess of DG and releasing it later when constraints are not binding [4]. Storage facilities could also be controlled to absorb or inject reactive power within the rating of the power conversion system, allowing further flexibility to manage voltages [5].

This work was supported in part by the University of Jordan, Jordan, and the EPSRC project WISE PV (Grant EP/K022229/1).

The authors are with the School of Electrical and Electronic Engineering, The University of Manchester, Manchester M13 9PL, UK. (email: sahban.alnaser@postgrad.manchester.ac.uk, luis_ochoa@ieee.org)
One of the significant challenges in the adoption of storage facilities, similar to other assets, is its appropriate sizing. Furthermore, given the ANM context in which these facilities will be used, sizing approaches need to integrate near realtime operational aspects to capture the actual power and energy needs of storage [6-8].

In the literature, sizing of storage in the context of active distribution networks and DG has been proposed mainly adopting trial and error approaches. In [9-11] this is done by investigating a number of predetermined storage sizes considering different control schemes. The 'optimal' size is the one achieving a trade-off between the cost of the storage facility and the operational cost [9-10] or the volume of curtailment [11]. Although trial and error approaches might seem implementable and practical, they may need to explore a large search space due to the combinations of power and energy capacities. Adequately covering this search space becomes even more challenging when multiple storage facilities are considered simultaneously.

Storage sizing techniques based on classical and metaheuristic optimization approaches were proposed in [12-13] (AC optimal power flow - OPF) and [14] (genetic algorithm), allowing a more thorough exploration of the corresponding search space. In [12], although multiple storage facilities are considered, the aggregated capacity is sized for the required daily wind energy curtailment. This approach neglects the discharge periods that, if adequately taken into account, might reduce the required capacities of storage facilities. Another way of sizing these facilities is by considering the corresponding investment cost in the context of the provision of services (i.e., for profit or savings). In [13] and [14], the capital cost of multiple storage facilities was incorporated as well as potential revenue streams but renewable energy curtailment was not part of the optimization.

Most of the above studies [9, 12-14] consider demand and generation on an hourly basis, neglecting near real-time changes that can be exploited by charge and discharge interactions for a more accurate sizing of storage. In addition, hourly intervals (or even 30 or 15-min [10-11]) are not adequate to model the actual operation of other controllable network elements (e.g., on-load tap changer) that have the potential to bring further benefits in the context of ANM.

To properly address the challenges described above this work presents an innovative optimization framework using a two-stage approach by which real-time control aspects and multiple storage facilities can be taken into account. The first stage uses a multi-period AC Optimal Power Flow (OPF) 
across the studied horizon considering simplified hourly wind and load profiles. By limiting the search space, this stage is capable of obtaining initial storage sizes (power and energy). The second stage adopts a high granularity minute-by-minute control driven by a mono-period bi-level AC OPF. This control minimizes the required curtailment using the firststage storage sizes so the actual curtailment can be assessed over the studied horizon. This assessment is then used to tune the first-stage storage sizes accordingly. Congestion and voltages are managed through the intelligent control of storage (active and reactive power), on-load tap changers (OLTCs), DG power factor, and DG curtailment as last resort. The proposed storage planning framework is applied to a real $33 \mathrm{kV}$ network from the North West of England to which wind farms and storage sites are added. One-minute resolution wind and load profiles for the first week of February 2010 are used.

The rest of this paper is structured as follows: section II presents the planning framework for sizing storage facilities. The formulation is explained in section III. The proposed approach is applied on a $33 \mathrm{kV}$ network incorporating multiple wind farms and storage facilities. The case studies are briefly described in section IV. Section V and VI demonstrate the proposed approach for single and multiple storage facilities, respectively. The potential integrated planning of DG plants and storage is briefly discussed in section VII. Finally, conclusions are drawn in section VIII.

\section{StORAGE Sizing FrameWORK}

To assess the potential benefits of storage facilities on the reduction of wind power curtailment (resulting from the management of congestion or voltage issues), an adequate horizon has to be considered (e.g., a week, a month, a year). Furthermore, given that the ability of storage to charge/discharge energy depends on previous control actions, it is necessary to consider the time-series nature of load and generation present in the studied network. Hence, the complexity of finding optimal storage sizes for a given horizon increases with the granularity of the time-series data.

The proposed framework to find the minimum storage size (power and energy) for a desired curtailment level (\% of the capacity factor) is presented in Fig. 1. The adoption of two stages, planning and control, reduces the computational burden that would otherwise be created by incorporating high granularity intervals in the first stage. Each of the stages and the iterative procedure are described below.

\section{A. First Stage (Planning)}

The multi-period AC OPF proposed in [2], formulated without considering the time-dependency between consecutive periods, is modified to cater for the inter-temporal constraints of storage facilities. For this purpose, the proposed formulation keeps track of the stored energy within each period of the studied time-series data so that the most suitable actions can be taken (more details in section III).

By minimizing the total cost of storage, this OPF finds the minimum sizes (MVA, MWh) able to reduce curtailment below a desired level. This is done considering all the time

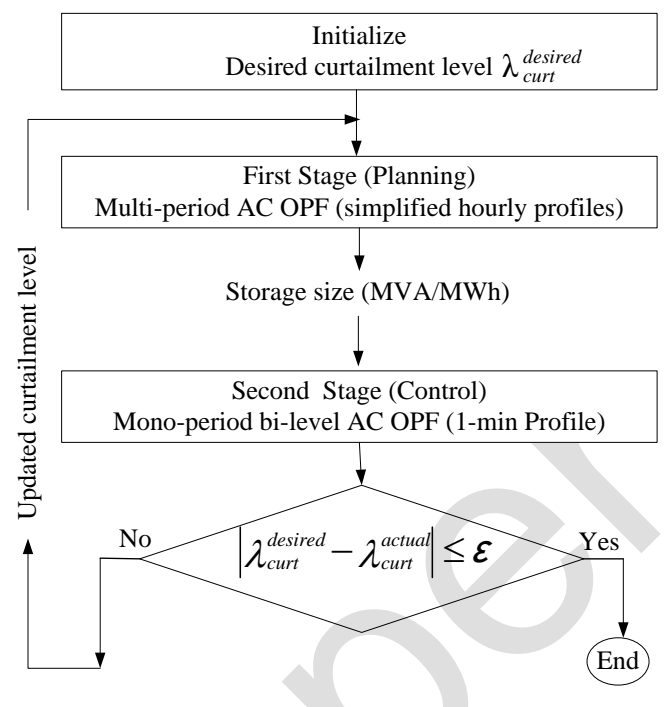

Fig. 1 Two-stage storage sizing framework.

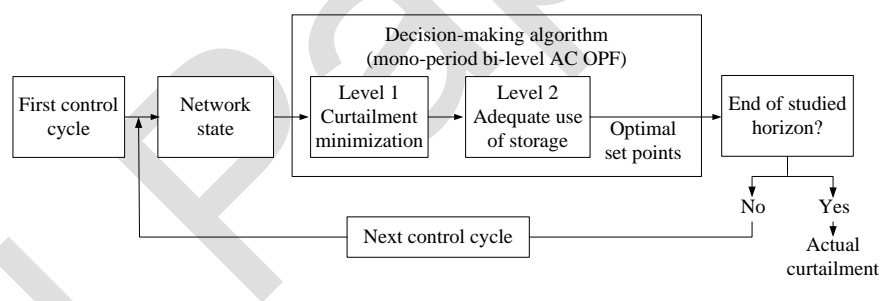

Fig. 2 Second stage (control) framework.

steps/periods within the studied horizon simultaneously.

The OPF also embeds ANM schemes that provide further flexibility to manage congestion and voltage constraints, potentially allowing the use of smaller storage sizes. This includes the control of tap positions of OLTCs as well as the power factor of DG plants and storage facilities.

This first stage considers hourly time-series data as a tradeoff between the scale of the problem and the high granularity representation of the operational aspects. However, this granularity might result in over or undersized storage facilities given that intra-hourly fluctuations have been neglected. For instance, the storage requirements for a congestion issue due to a 60-min average generation can be significantly different from those resulting from 15 or 1 minute values where sudden gusts of wind can occur. Consequently, to check the effectiveness of the storage sizes found by the first stage, a second stage is introduced by which the optimal control is applied adopting a high granularity control environment.

\section{B. Second Stage (Control)}

The second stage examines the actual curtailment level to be achieved by the storage sizes determined in the first stage. This is done by considering 1-minute control cycles. As shown in Fig. 2, at the start of each control cycle, the states of loads, generation, storage devices and OLTCs' are sent to the decision-making algorithm to find the best set points of controllable elements that allow minimizing generation curtailment (Level 1). To guarantee that the storage devices only store the excess of generation determined in Level 1, Level 2 is introduced. In addition, for those control cycles where there is no need of curtailment, Level 2 also determines 
the adequate level of discharge. After Level 2, the final optimal set points for all the controllable elements are issued.

The decision-making algorithm is formulated as a monoperiod bi-level AC OPF. It is mono-period because it only deals with a single network state. It is bi-level because the optimization carried out in Level 2 requires the curtailment determined by the optimization in Level 1.

The volume of curtailment resulting from each control cycle is aggregated throughout the studied horizon to determine the actual curtailment level achieved by the corresponding storage sizes.

\section{Iterative Procedure}

If the difference between the actual curtailment level $\lambda_{\text {curt }}^{\text {actual }}$ obtained at the second stage and the desired curtailment level $\lambda_{\text {curt }}^{\text {desired }}$ is outside the defined tolerance $\varepsilon$ (see Fig. 1), new storage sizes are required. For this, the first stage is given an updated curtailment level which is calculated by adjusting the previous curtailment level up or down according to the binding tolerance and a defined step.

For example, if the actual curtailment at the second stage is found higher than the desired one, the curtailment level from the last iteration is reduced by the defined step (e.g., 1\%). This process is iteratively applied until the difference between the actual curtailment at the second stage and the desired one falls within the tolerance.

\section{PROBLEM Formulation}

This section presents the formulations of the proposed framework aimed at finding the minimum energy capacity $E_{s t}^{\text {rated }}(\mathrm{MWh})$ and apparent power rating $S_{s t}^{\text {rated }}(\mathrm{MVA})$ of storage facilities (set $S T$ indexed by $s t$ ) required to achieve the desired curtailment level, $\lambda_{\text {curt }}^{\text {desired }}$. The section is divided into planning and control stages, and the iterative procedure.

\section{A. Planning Stage "Multi-Period AC OPF"}

The multi-period AC OPF with inter-temporal constraints considers for each period (set $T$ indexed by $t$ ) the hourly average of the high granularity wind and demand profiles.

To determine the minimum size of storage facilities, for both energy and power, the objective function is formulated to minimize a proxy of the overall capital cost. This allows realistically relating energy capacities and power ratings in a single equation, as shown in (1).

$$
\min \sum_{s t \in S T} C_{E} E_{s t}^{\text {rated }}+C_{S} S_{s t}^{\text {rated }}
$$

The weighting coefficients $C_{E}$ and $C_{S}$ are the relative costs of the energy capacity and the apparent power rating, respectively. Here, the proportion between these weighting coefficients is used instead of their actual values. In particular, the cost of the energy capacity is considered to be 1.5 times that of the apparent power rating, i.e., $C_{E}=1$ unit cost per MWh and $C_{S}=0.67$ unit cost per MVA, corresponding to a LithiumIon battery [15].

This objective is subject to a range of constraints including those related to the storage facilities, controllable DG plants and OLTCs, voltage and thermal limits, as well as the traditional AC OPF constraints (i.e., Kirchhoff's voltage law and power balance).

\section{1) Storage facilities}

Storage facilities are controlled to discharge (i.e., inject) or charge (i.e., absorb) active power, $p_{s t, t}$, at each time step within the converter apparent power rating, $S_{s t}^{\text {rated }}$. Positive values of $p_{s t, t}$ correspond to injection of power (i.e., discharging).

$$
-S_{s t}^{\text {rated }} \leq p_{s t, t} \leq S_{s t}^{\text {rated }} ; \forall s t, t
$$

It is worth noting that depending on the storage technology different power limits can exist for charging and discharging. In addition, these limits can also vary depending on the level of stored energy. Equation (2) can be modified and expanded by having different limits per level of stored energy.

It is important to highlight that this approach of adopting a single variable to model power injection/absorption is an improvement from previous studies [10-12, 16] where two variables were used to represent charging and discharging. In those cases, to ensure that charging and discharging does not occur simultaneously (due to the two variables), it was considered: a Mixed Integer Non-Linear Programming (MINLP) formulation [16], the adoption of pre-determined charge/discharge periods [11-12], and the use of the net power from charging and discharging (which overestimates conversion losses) [10]. Therefore, the proposed singlevariable formulation is potentially more scalable (as larger problems can be solved using a non-linear programming formulation) and general (since scheduling or net power approaches are not needed).

The energy losses that result from energy and power conversion have to be accounted for during charging and discharging. Therefore, the change in the stored energy, $\Delta E_{s t, t}^{\text {stor }}$, at each time step and the corresponding stored energy, $E_{s t, t}^{\text {stor }}$, can be represented by (3) and (4), respectively.

$$
\begin{array}{ll}
\Delta E_{s t, t}^{\text {stor }}=\left\{\begin{array}{ll}
-\frac{p_{s t, t} \Delta t}{\eta_{s t}^{\text {dis }}}, & p_{s t, t} \geq 0 \\
-p_{s t, t} \eta_{s t}^{c h} \Delta t, & p_{s t, t}<0
\end{array}\right\} & \forall s t, t \\
E_{s t, t}^{\text {stor }}=E_{s t}^{(0)}+\sum_{t=1}^{t} \Delta E_{s t, t}^{\text {stor }} & \forall s t, t
\end{array}
$$

Where $\eta_{s t}^{c h}$ and $\eta_{s t}^{\text {dis }}$ are the charging and discharging efficiencies, respectively. $\Delta t$ is 1 hour and $E_{s t}^{(0)}$ is the initial stored energy at the beginning of the planning horizon.

To preserve the lifetime of battery storage facilities, the stored energy can be kept above a particular percentage of the capacity [17]. This can be expressed as the maximum Depth of Discharge $\left(D o D^{\max }\right)$. Therefore, the stored energy is controlled between a minimum level and its rated capacity.

$$
\left(1-D o D^{\text {max }}\right) E_{\text {st }}^{\text {rated }} \leq E_{\text {st }, t}^{\text {stor }} \leq E_{\text {st }}^{\text {rated }} ; \forall s t, t
$$

Finally, each storage facility is also controlled to inject or absorb reactive power $q_{s t, t}$ such that the apparent power output at each period within the planning horizon does not exceed the corresponding apparent power rating $S_{s t}^{\text {rated }}$.

$$
\left(p_{s t, t}\right)^{2}+\left(q_{s t, t}\right)^{2} \leq\left(S_{s t}^{\text {rated }}\right)^{2} ; \forall s t, t
$$

The power factor angle of storage, $\emptyset_{s t, t}$, is used to determine the reactive power and must be within the 
corresponding capability range of the device, $\emptyset_{s t}^{(+,-)}$.

$$
\begin{aligned}
& q_{s t, t}=p_{s t, t} \tan \left(\emptyset_{s t, t}\right) \\
& \emptyset_{s t}^{-} \leq \emptyset_{s t, t} \leq \emptyset_{s t}^{+} ; \forall s t, t
\end{aligned}
$$

\section{2) Controllable DG plants (Curtailment)}

The controllable DG plants (set $N$ indexed by $n$ ) can be subject to curtailment to keep voltage and thermal constraints within limits. The power output, $p_{n, t}$, at each time step is limited by the difference of the available power resource

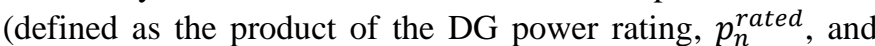
the normalized wind power pattern, $\Gamma_{t}$ ) and the curtailed power, $p_{n, t}^{\text {curt }}$, for the same period. This is presented in (9).

$$
p_{n, t}=p_{n}^{\text {rated }} \Gamma_{t}-p_{n, t}^{\text {curt }} ; \forall n, t
$$

However, the total volume of curtailment for all DG plants will be limited to the curtailment level, $\lambda_{\text {curt }}^{v}$ (at iteration $v$ ). This is a percentage of the total available energy resource that could be harvested across the planning horizon.

$$
\sum_{n \in N, t \in T} p_{n, t}^{\text {curt }} \leq \lambda_{\text {curt }}^{v} \sum_{n \in N, t \in T} p_{n}^{\text {rated }} \Gamma_{t}
$$

Furthermore, the controllable DG plants can operate at different power factor angles, $\emptyset_{n, t}$. This makes reactive power "dispatchable" within the MVA capability, $S_{n}^{\text {rated }}$, of the corresponding DG plant. This angle, however, must be within the allowable power factor angle range $\emptyset_{n}^{(-,+)}$.

$$
\begin{gathered}
\left(p_{n, t}\right)^{2}+\left(p_{n, t} \tan \left(\emptyset_{n, t}\right)\right)^{2} \leq\left(S_{n}^{\text {rated }}\right)^{2} ; \forall n, t \\
\emptyset_{n}^{-} \leq \emptyset_{n, t} \leq \emptyset_{n}^{+} ; \forall n, t
\end{gathered}
$$

\section{3) On-Load Tap Changers}

Lines and transformers are all modelled within the same set (set $L$ indexed by $l$ ). The type of each element in this set is determined using an integer parameter, $\alpha_{l}$, whose values are zero for lines, 1 for OLTC-fitted transformers, and 2 for offload tap changing transformers. The tap ratio, $\tau_{l, t}$, for OLTCfitted transformers $\left(\alpha_{l}=1\right)$ can be controlled at each time step considering its capabilities $\tau_{l}^{(-,+)}$.

$$
\tau_{l}^{-} \leq \tau_{l, t} \leq \tau_{l}^{+} ; \forall l, t
$$

In contrast, tap ratios for off-load tap changing transformers $\left(\alpha_{l}=2\right)$ are not controlled and they are inputs to the optimization problem.

\section{4) General AC OPF constraints}

Active, $f_{l, t}^{(1,2), P}$, and reactive power, $f_{l, t}^{(1,2), Q}$, injections at the start and end buses (denoted 1 and 2) for each line and each transformer are calculated according to the standard Kirchhoff's voltage law expressions as presented below [2].

$$
\begin{aligned}
& f_{l, t}^{(1,2), P}=f_{l, t, K V L}^{(1,2), P}(\boldsymbol{V}, \boldsymbol{\delta}) ; \forall l, t \\
& f_{l, t}^{(1,2), Q}=f_{l, t, K V L}^{(1,2), Q}(\boldsymbol{V}, \boldsymbol{\delta}) ; \forall l, t
\end{aligned}
$$

If the transformers are equipped with OLTCs $\left(\alpha_{l}=1\right)$, the corresponding terms in (14) and (15) for voltage at the start bus of the line will be divided by the tap ratio $\tau_{l, t}$.

The distribution network is supplied by at least one point interfacing with the upstream grid. It is assumed that this point $x$ ( $X$, set of external connections) can import/export real and reactive power $\left(p_{x, t}, q_{x, t}\right)$ within limits $\left(p_{x}^{(-,+)}, q_{x}^{(-,+)}\right)$. One of these external connections is taken as the slack bus with voltage angle, $\delta_{\text {slack }, k}$, equal to zero.
The balance of real and reactive power at each bus (set $B$ indexed $b$ ), and for each time step, is formulated according to (16) and (17), respectively. The modeling considers firm generation (set $G$ indexed by $g$ ), whose power output, $p_{g, t}$, not controllable. They are assumed to have fixed power factor, $\emptyset_{g}$.

$$
\begin{aligned}
& \sum_{n \in N \mid \beta_{n=b}} p_{n, t}+ \sum_{g \in G \mid \beta_{g=b}} p_{g, t}+\sum_{x \in X \mid \beta_{x=b}} p_{x, t} \\
&+\sum_{s t \in S T \mid \beta_{s t=b}} p_{s t, t} \\
&=d_{b, t}^{P}+\sum_{n \in N \mid \beta_{n=b}} f_{l, t}^{(1,2), P} ; \forall l, t \\
& \sum_{l \in L \mid \beta_{l}^{(1,2)}=b} \tan \left(\emptyset_{n, t}\right)+\sum_{g \in G \mid \beta_{g=b}} p_{g, t} \tan \left(\emptyset_{g}\right) \\
&+\sum_{x \in X \mid \beta_{x=b}} q_{x, t} \\
&+\sum_{s t \in S T \mid \beta_{s t=b}} p_{s t, t} \tan \left(\emptyset_{s t, t}\right) \\
&=d_{b, t}^{Q}+\sum_{l \in L \mid \beta_{l}^{(1,2)}=b} f_{l, t}^{(1,2), Q} ; \forall l, t
\end{aligned}
$$

where $\beta_{u}$ maps the location of each network element ( $u \subset$ $\{s t, n, g, x, l\})$ to its associated bus, $b$ and, $d_{b, t}^{(P, Q)}$ are the active and reactive demand at the same bus.

The voltage magnitude at bus $b\left(V_{b, t}\right)$ should be within the statutory limits, $V_{b}^{(-,+)}$, and the apparent start/end power flows through lines and transformers should be limited to their corresponding thermal capacity $f_{l}^{+}$.

$$
\begin{gathered}
V_{b}^{-} \leq V_{b, t} \leq V_{b}^{+} ; \forall b, t \\
\left(f_{l, t}^{(1,2), P}\right)^{2}+\left(f_{l, t}^{(1,2), Q}\right)^{2} \leq\left(f_{l}^{+}\right)^{2} ; \forall l, t
\end{gathered}
$$

\section{B. Control Stage "Mono-Period Bi-Level AC OPF"}

This stage finds the actual curtailment level, $\lambda_{\text {curt }}^{\text {actual }}$, that could be achieved by the storage sizes obtained in the first stage $\left(S_{s t}^{\text {rated }}, E_{s t}^{\text {rated }}\right)$ considering 1-min control cycles.

The optimization carried out in Level 1 maximizes the total active power of the controllable DG plants, $p_{n}$, whose maximum values are restricted by the available resource, $p_{n}^{+}$, at that control cycle. By maximizing the harvesting of DG plants, the minimum volume of curtailment is ensured for the corresponding control cycle.

$$
\begin{gathered}
\max \sum_{n \in N} p_{n} \\
p_{n} \leq p_{n}^{+}
\end{gathered}
$$

where the available power resource $p_{n}^{+}$is product of the DG power rating, $p_{n}^{\text {rated }}$, and the normalized wind power pattern, $\Gamma_{t}$, for that control cycle.

This objective function is also subject to those constraints in the first stage related to the storage facilities (2), (5)-(8), the controllable DG units (11)-(12), the controllable OLTCs (13), and, the general AC OPF constraints (14)-(19). These constraints are adapted to consider a single time period. In particular, similar to [3], the near real-time operation of locally controlled OLTCs is also modeled. To cater for this within the 
optimization, the values of the tap positions are considered as fixed parameters $\left(\alpha_{l}=2\right)$.

In addition, for each control cycle, $T c$, the energy stored at the previous one, $E_{s t}^{T c-1}$, is used as initial condition to find the new store level, $E_{s t}^{T c}$, as given in (22).

$$
E_{s t}^{T c}=\left\{\begin{array}{cc}
E_{s t}^{T c-1}-\frac{p_{s t} \Delta T_{c}}{\eta_{s t}^{d i s}}, & p_{s t} \geq 0 \\
E_{s t}^{T c-1}-p_{s t} \eta_{s t}^{c h} \Delta T_{c}, & p_{s t}<0
\end{array}\right\} ; \forall s t
$$

where $\Delta T_{c}$ is $1 / 60$ hour (i.e., 1-min control cycle).

Once the maximum active power of controllable DG is identified, the volume of curtailment $E_{\text {curt }}$ can be determined.

$$
E_{\text {curt }}=\sum_{n \in N}\left(p_{n}^{+}-p_{n}\right) \Delta T_{c}
$$

This volume of curtailment is then used by the optimization in Level 2 to ensure that the storage facilities are adequately used for the corresponding control cycle (see Section II.B). To do so, Level 2 minimizes the stored energy, (24), subject to all constraints in Level 1 plus one further constraint, (25). The latter guarantees that curtailment will not go beyond the one previously calculated $\left(E_{\text {curt }}\right)$.

$$
\begin{gathered}
\min \sum_{s t \in S T} E_{s t}^{T c} \\
\sum_{n \in N}\left(p_{n}^{+}-p_{n}\right) \Delta T_{c} \leq E_{\text {curt }}
\end{gathered}
$$

The output of Level 2 corresponds to the final set points for all controllable elements within the analyzed control cycle.

At the end of the studied horizon, the actual curtailment level, $\lambda_{\text {curt }}^{\text {actual }}$, can be calculated by adding up the curtailment volumes of each cycle, $E_{\text {curt }}$, and dividing the total by the horizon's available energy resource.

It is worth highlighting that this stage considers an ideal control action, i.e., decisions and actions happen within the same control cycle. This eliminates the potential noise created by the changes in demand and generation from one minute to another, resulting in a much cleaner quantification of the benefits from storage facilities in the context of renewable DG curtailment minimization.

Finally, given that the tap ratios of centrally controlled OLTCs are modelled by the mono-period bi-level optimization as continuous variables (13), the values should be converted to the nearest discrete ones that reflect a feasible integer tap position (similarly to the procedure presented in [3]). Once all the optimal set points are found, they are applied in a distribution network analysis software to allow the adequate operation of the locally controlled OLTCs. This is important as the resulting tap positions will be used as inputs for the optimization in the next time step and thus can affect the calculation of the actual curtailment level.

\section{Iterative Procedure}

Further iterations may be needed to produce new sizes of storage facilities until the difference between the actual curtailment level and the desired curtailment level becomes smaller than the defined tolerance, $\varepsilon$.

$$
\left|\lambda_{\text {curt }}^{\text {actual }}-\lambda_{\text {curt }}^{\text {desired }}\right| \leq \varepsilon
$$

The curtailment level, $\lambda_{\text {curt }}^{v}$, used by the first stage is given by (27) in each iteration $v$. For the first iteration $(v=1)$, this curtailment level is set to the desired curtailment level, $\lambda_{\text {curt }}^{\text {desired }}$. Then, in the subsequent iterations $(v>1)$, it will be modified by the step, $\Delta^{v}$, from its previous value, $\lambda_{\text {curt }}^{v-1}$.

$$
\begin{gathered}
v=1 \\
v>1 \quad\left\{\begin{array}{c}
\lambda_{\text {curt }}^{v}=\lambda_{\text {curt }}^{\text {desired }}=\lambda_{\text {curt }}^{v-1}-\Delta^{v} ; \lambda_{\text {curt }}^{\text {actul }}-\lambda_{\text {curt }}^{\text {desired }}>0 \\
\lambda_{\text {curt }}^{v}=\lambda_{\text {curt }}^{v-1}+\Delta^{v} ; \lambda_{\text {curt }}^{\text {actual }}-\lambda_{\text {curt }}^{\text {desired }}<0
\end{array}\right.
\end{gathered}
$$

Note that $\lambda_{\text {curt }}^{v}$ is only used to force the planning stage to improve the storage sizes. Ultimately, a value of $\lambda_{\text {curt }}^{\text {actual }}$ within $\varepsilon$ of the desired value is the target of the iterative procedure.

\section{REAL UK MV NETWORK AND MODELLING}

The proposed storage-sizing framework is applied in sections V and VI to a real UK $33 \mathrm{kV}$ network from the North West of England. The single-line representation of the network is given in Fig. 3 [3]. The target voltage for the OLTC at the Bulk Supply Point (BSP) is set to 1.02p.u.. The network initially has four DG plants (in Fig. 3) with firm connection agreements (i.e., not controllable) at buses 201, 205, 206 and 210 with capacities of 9.1, 10.6, 12 and 7.5 MW, respectively (total capacity of $39.2 \mathrm{MW}$ ). The maximum and the minimum demands for the whole network are 31 and 12 MW, respectively. Half-hourly load profiles at each primary substation are based on the corresponding number and type of customers. To carry out 1-min resolution analyses, these halfhourly profiles persists over the corresponding 30-minute period. Minute-by-minute wind profiles for the first week of February 2010 are adopted for all the DG plants. This week corresponds to the one with the highest wind capacity factor in February and therefore provides a challenging scenario for the sizing and control of storage facilities.

The modeling language AIMMS [18] is used to formulate and solve the multi-period AC OPF (planning stage) as well as the mono-period bi-level AC OPF (control stage) and solved using the CONOPT $3.14 \mathrm{~V}$ NLP solver. The distribution network analysis software package OpenDSS is used at the control stage to provide the network state at each control cycle before and after applying the optimal set points [19].

\section{CASe Study: Single Storage Facility}

In this section, the benefits of optimally operating storage facilities in the context of curtailment reduction are demonstrated considering a single storage facility at bus 201 to manage congestion issues in line 200-201 due to two new DG plants. The effects of data granularity on storage sizing are also investigated. Finally, the two-stage approach is demonstrated by finding the optimal size of storage.

\section{A. No Storage - Optimal Curtailment Only}

A new 9 MW wind power plant is connected as firm generation to bus 201. This corresponds to the maximum capacity that can be connected to this location without overloading line 200-201 (20 MVA) during minimum load. Furthermore, a $6 \mathrm{MW}$ controllable DG plant is connected to bus 201. By controlling the active power of the 6 MW DG 


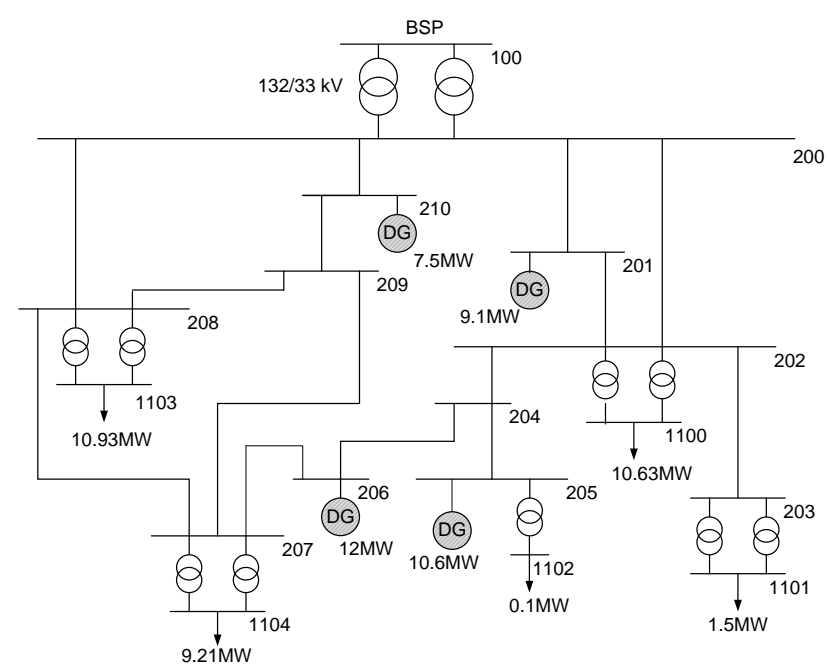

Fig. 3 Real UK $33 \mathrm{kV}$ network from the North West of England.

plant it is possible to mitigate the overloads through line 200201. To illustrate the volume of curtailment required to do so, the minute-by-minute wind profile corresponding to $1^{\text {st }}$ February 2010 is adopted for all DG plants. The Level 1 mono-period $\mathrm{AC} \mathrm{OPF}$ is used to calculate the curtailment needed to avoid congestions at each minute. The resulting curtailment is $51 \mathrm{MWh}$, i.e., $42 \%$ of the available resource $(121.7 \mathrm{MWh})$, and the maximum power curtailed at any minute is $5.9 \mathrm{MW}$

\section{B. Operation of Storage (Bi-Level Mono-period AC OPF)}

This section demonstrates the benefits of the bi-level monoperiod AC OPF to optimally control the operation of a storage facility (charging, discharging and idling) at bus 201 and the 6 MW DG plant. Similarly to section V-A, this is carried out using a realistic control cycle length of 1 minute.

A lithium-ion storage facility will be used given the required fast response. It is considered to have round trip efficiency of $85 \%$, i.e., charging $\left(\eta_{s t}^{c h}\right)$ and discharging $\left(\eta_{s t}^{d i s}\right)$ efficiencies of $92 \%$. The depth of discharge is permitted to reach $80 \%$ of the energy capacity $\left(D o D^{\max }=80 \%\right)$ [15].

To demonstrate the benefits of the proposed approach, in particular the control aspects, an initial arbitrary size of 4 MVA and $12 \mathrm{MWh}$ is considered for the battery at 201. To clearly illustrate the operation of the control stage in this case study, only the active power of storage is controlled and its power factor is kept at unity (i.e., no reactive power).

To illustrate the optimal operation of storage, the power flow through line 200-201 that would occur without controlling the $6 \mathrm{MW}$ DG plant and the storage facility is presented in Fig. 4. Parallel to this, Fig. 5 presents the percentage of harvested wind power (red line) and the stored energy in MWh (blue line) found by the optimization in each control cycle, i.e., resulting in no congestion issue. The harvested wind power using the "curtailment-only" scheme (black line) is also presented to show the benefits of storage in the reduction of curtailment.

At the beginning of the day, from 0:00 to 1:40, due to the excess of generation (see Fig. 4), the storage is required to deal with the congestion of line 200-201. However, throughout this period, the excess power is larger than the storage rating (4 MVA), resulting in curtailment (harvested energy less than $100 \%$ ). For those periods where the excess generation is less than 4 MVA, such as 4:00 to 10:00, the storage is capable of managing the congestion issue without the need of curtailment.

To ensure storage capacity is available for future congestion events, discharging has to be applied when there is enough headroom in line 200-201. For instance, from 1:40 to 3:00, although there are periods with excess generation, there are also those with sufficient headroom to allow the discharging. This process lasts until the stored energy reaches the minimum of $2.4 \mathrm{MWh}$ according to the adopted $D o D^{\max }$ of $80 \%$ (i.e., $0.2 \times 12 \mathrm{MWh})$. In addition, the storage goes into idling mode when there is no need of charging (e.g., 9:00 to 10:00) or no possibility of discharging (e.g., 19:00 to 21:00). Indeed, once the stored energy reaches the storage capacity (12 MWh), curtailment will be the only solution to manage congestion.

The resulting curtailment level using the optimal operation of storage and the $6 \mathrm{MW}$ DG plant is $24 \%(29.2 \mathrm{MWh})-\mathrm{a}$ significant reduction from the $42 \%$ found by applying only curtailment.

\section{Effects of Data Granularity on Storage Sizing}

The proposed mono-period bi-level AC OPF can also be used to investigate the effects of data granularity on storage sizing. This is of particular importance given that most studies found in the literature adopt hourly data [9, 12-14].

A comparison is carried out considering 1 and 60-minute resolution profiles as well as different sizes for a storage facility located at bus 201 . The required level of curtailment is used as the performance metric. The 60-min wind and load profiles are obtained by averaging the 1-min profiles previously used. The adopted profiles are presented in Fig. 6 .

Six sizes for the storage facility are analyzed. The power rating is selected from $1 \mathrm{MW}$ to $6 \mathrm{MW}$ (maximum curtailment required, see Fig. 4). For illustration purposes, the corresponding energy capacity is considered to be three times the power rating.

The curtailment levels for each storage size and data granularity case are presented in Table I. It can be seen that curtailment is always underestimated using hourly profiles given that issues will need to be solved at shorter intervals.

This also can have a significant impact when deciding the most cost-effective storage size. For example, using hourly profiles, a storage facility of $2 \mathrm{MVA}$ and $6 \mathrm{MWh}$ seems to be capable of limiting curtailment to $27 \%$. However, the more accurate 1-min case shows that $27 \%$ can only be achieved with a 3 MVA and 9 MWh storage facility.

The misleading curtailment achieved when adopting hourly profiles are due to the fact that the intra-hour fluctuations -and the corresponding congestion issues- are neglected. This can be observed from the optimal operation of the 2 MVA and 6 MWh storage and the $6 \mathrm{MW}$ controllable DG plant presented in Fig. 7. For example, for the hourly profile between 2:00 and 11:00 there is no congestion issue and therefore the storage is at idling mode and the DG unit is not curtailed. However, due 


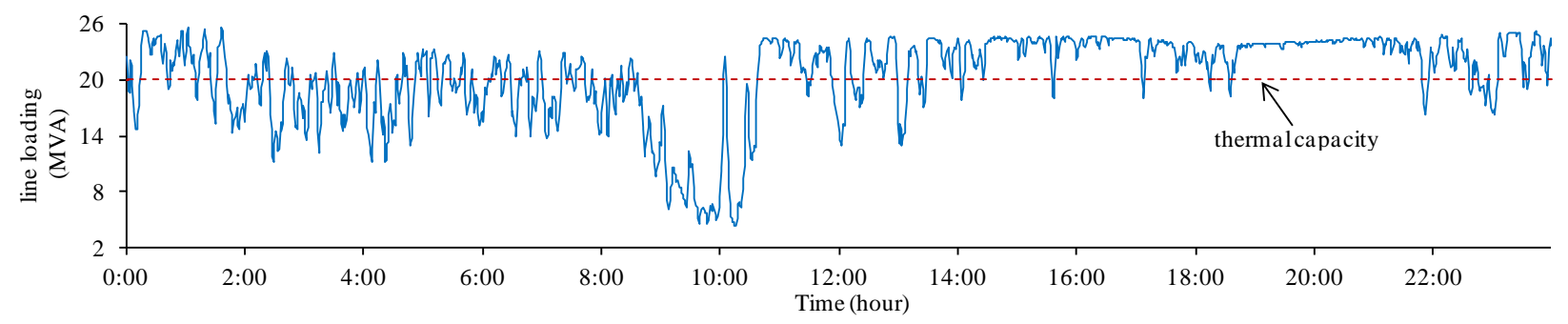

Fig. 4 Power flow through line 200-201 (MVA) without controlling the 6 MW DG plant and no storage $-1^{\text {st }}$ February 2010.

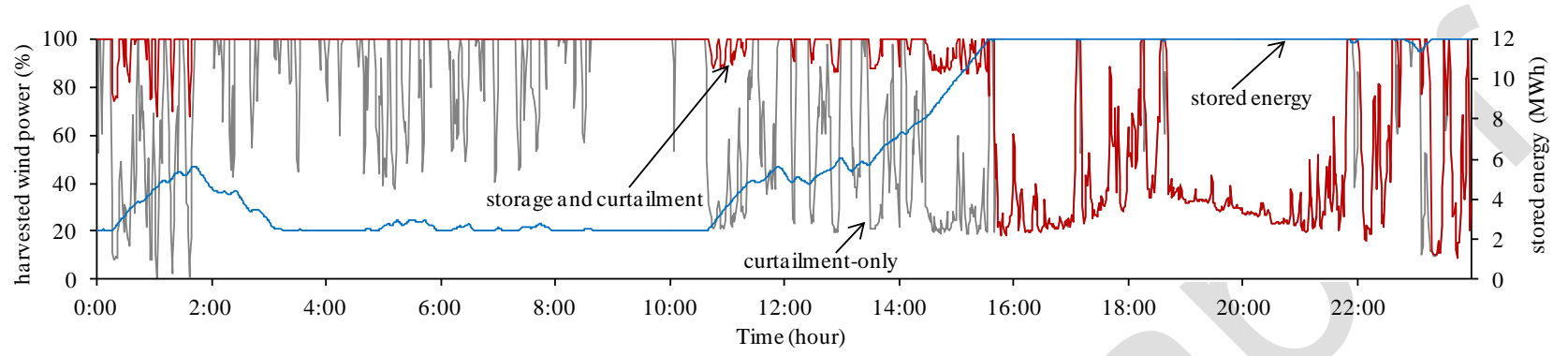

Fig. 5 Harvested wind power from the 6 MW DG plant (in \%) and stored energy (MWh) for the 4 MVA-12 MWh storage facility at bus $201-1^{\text {st }}$ February 2010.

TABLE I

Curtailment for Different Sizes of Storage and Data Granularity $-1^{\text {st }}$ Feb

\begin{tabular}{c|c|c|c|c}
\hline \multicolumn{2}{c|}{ Storage size } & \multicolumn{3}{c}{ Curtailment $(\%)$} \\
\hline MVA & MWh & $60-$ min & 1-min & Error \\
\hline- & - & 33.0 & 42.0 & 9.0 \\
\hline 1 & 3 & 29.8 & 35.8 & 6.0 \\
\hline 2 & 6 & 26.8 & 30.9 & 4.1 \\
\hline 3 & 9 & 24.5 & 27.1 & 2.6 \\
\hline 4 & 12 & 22.3 & 24.1 & 1.8 \\
\hline 5 & 15 & 20.2 & 21.4 & 1.2 \\
\hline 6 & 18 & 18.1 & 18.9 & 0.8 \\
\hline
\end{tabular}

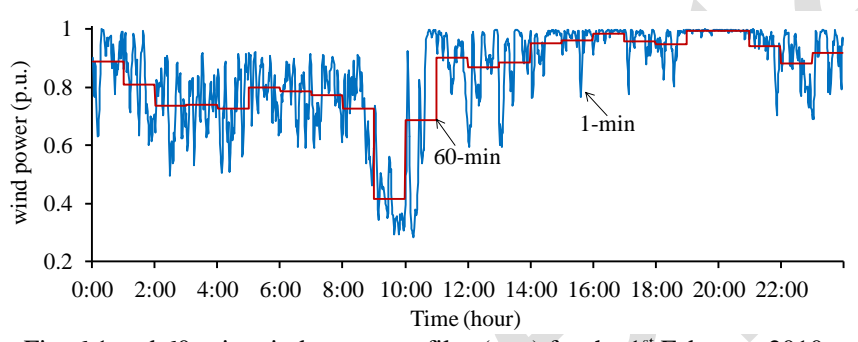

Fig. 61 and 60-min wind power profiles (p.u.) for the $1^{\text {st }}$ February 2010.

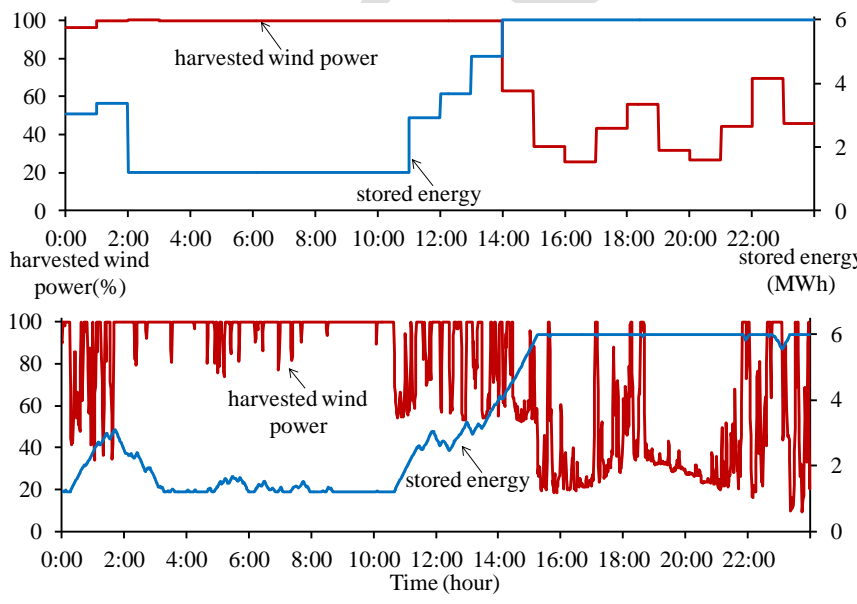

Fig. 7 Harvested wind power from the 6 MW DG plant (in \%) and stored energy (MWh) for the 2 MVA-6 MWh storage facility for the $1^{\text {st }}$ February 2010; (top) 60-min and (bottom) 1-min resolution profiles.
TABLE II

Curtailment for Different Sizes of Storage and Data Granularity $-2{ }^{\text {nd }} F e b$

\begin{tabular}{c|c|c|c|c}
\hline \multicolumn{2}{c|}{ Storage size } & \multicolumn{3}{c}{ Curtailment $(\%)$} \\
\hline MVA & MWh & $60-$ min & 1-min & Error \\
\hline- & - & 52.2 & 57.0 & 4.8 \\
\hline 1 & 3 & 48.0 & 52.3 & 4.3 \\
\hline 2 & 6 & 45.0 & 48.2 & 3.2 \\
\hline 3 & 9 & 42.2 & 44.7 & 2.5 \\
\hline 4 & 12 & 40.1 & 42.7 & 2.6 \\
\hline 5 & 15 & 38.1 & 39.1 & 1.0 \\
\hline 6 & 18 & 36.1 & 36.7 & 0.6 \\
\hline
\end{tabular}

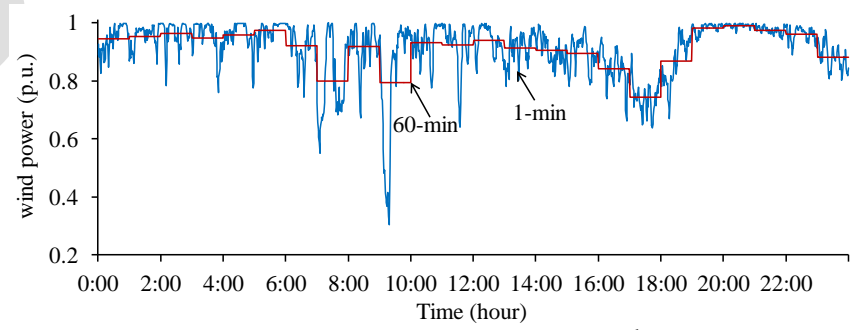

Fig. 81 and 60-min wind power profiles (p.u.) for the $2^{\text {nd }}$ February 2010.

to the intra-hour fluctuations of wind that will also affect the power output of firm generation (see Fig. 6), curtailment and storage of energy are in reality needed for the for the period between 2:00 and 11:00 when using 1-min resolution profiles.

It is interesting, however, that the difference in curtailment becomes smaller for larger storage sizes (Table I). Therefore, the use of simplified hourly profiles may be considered adequate when sizing storage in cases where significant reductions of curtailment are required. Nonetheless, it is still important to realistically model control aspects to truly quantify the curtailment level to be achieved.

To show the effects of a different wind power profile on the sizing of storage facilities, the above analysis is repeated using data for the $2^{\text {nd }}$ February (Fig. 8). The daily capacity factor is $91 \%$ (higher than the $85 \%$ of the day before). In addition, as it can be seen in Fig. 8, the minute-by-minute values are closer to the hourly averages than on $1^{\text {st }}$ February (Fig. 6). The curtailment levels for each storage size and data granularity 
TABLE III

Iterative Storage Sizing at Bus 201 for $21 \%$ desired curtailment

\begin{tabular}{c|c|c|c}
\hline \multirow{2}{*}{ Iteration } & $\begin{array}{c}\text { First Stage } \\
\text { Desired } \\
\text { curtailment }(\%)\end{array}$ & $\begin{array}{c}\text { Storage size } \\
\text { (MVA/MWh) }\end{array}$ & $\begin{array}{c}\text { Second Stage } \\
\text { Actual } \\
\text { curtailment (\%) }\end{array}$ \\
\hline 1 & 21 & $2.67 / 12.6$ & 24.9 \\
\hline 2 & 20 & $2.66 / 13.8$ & 24.0 \\
\hline 3 & 19 & $2.69 / 15.7$ & 22.7 \\
\hline 4 & 18 & $\mathbf{2 . 7 2 / 1 6 . 9}$ & $\mathbf{2 1 . 6}$ \\
\hline
\end{tabular}

are presented in Table II. The results show, as expected, that the hourly profile of $2^{\text {nd }}$ February performs better than that of the day before. Nonetheless, curtailment errors, and therefore storage sizing errors, still exist. These results also confirm that the curtailment error becomes smaller for larger storage sizes.

\section{Size of Storage "Two-Stage Iterative Framework"}

To find the storage size at bus 201 using the proposed twostage sizing framework (see Fig. 1) a desired curtailment level needs to be defined. A value of $21 \%$ is used to half the curtailment that would otherwise be required (section $\mathrm{V}-\mathrm{A}$ ). The tolerance is set to $+/-1 \%$ (i.e., the final actual curtailment level must be within 20 and $22 \%$ ) and the step to $1 \%$.

Table III presents the evolution of the sizing of the storage facility resulting from the iterative process including the corresponding actual curtailment.

After the first iteration, a storage size of 2.7 MVA and 12.6 MWh is suggested by the first stage (planning) to achieve the $21 \%$ desired curtailment. However, the second stage (control) reveals an actual curtailment of $24.9 \%$, much larger than the desired one. Thus, an updated curtailment level of $20 \%$ is triggered (as explained in section II-C). This iterative procedure stops at the fourth iteration resulting in a storage size of 2.7 MVA and 16.9 MWh which corresponds to an actual curtailment level of $21.6 \%$ (i.e., within the tolerance).

It can be noticed that through the iterative process the optimal power of storage changes only slightly. This is due to the persistence of periods with high wind generation (that eventually require curtailment) and the desired curtailment. Given that from 11:00 to midnight curtailment would be almost continuously required (see Fig. 4), the planning stage looks for larger energy capacities as it will be more beneficial to reach the desired curtailment at each iteration. However, if the desired curtailment were much tighter or looser, then the planning stage would also explore different power ratings.

It is important to highlight that simpler trial and error approaches (e.g., [9-11]) might be able to find comparable results for a single storage facility. However, the combination of other potential solutions such as the control of OLTCs and power factor (DG and storage) might not be possible given the increased number of options and complexity. This becomes a much larger issue if multiple storage sites are considered.

\section{Case Study: Multiple Storage Facilities}

This section investigates the two-stage sizing framework in a more complex environment considering multiple storage facilities and adopting different Active Network Management (ANM) schemes to increase the flexibility in solving congestion and voltages issues. The potential benefits from
TABLE IV

DG Plants Connected to the MV Network (Case Study VI)

\begin{tabular}{c|c|c|c}
\hline Bus & $\begin{array}{c}\text { Existing firm } \\
\text { generation (MW) }\end{array}$ & $\begin{array}{c}\text { New firm } \\
\text { generation (MW) }\end{array}$ & $\begin{array}{c}\text { New controllable } \\
\text { generation (MW) }\end{array}$ \\
\hline 201 & 9.1 & 6 & 3 \\
\hline 205 & 10.6 & 2 & 10 \\
\hline 206 & 12 & - & - \\
\hline 210 & 7.5 & 8 & 3 \\
\hline Total & 39 & 16 & 16 \\
\hline
\end{tabular}

incorporating further ANM schemes in the adoption of smaller storage capacities are also quantified. The studied horizon is extended for the first week of February 2010.

To achieve the maximum capacity that can be connected without voltage or congestion issues during minimum load, the capacities of firm wind generation (i.e., not controllable) in the real UK MV network (Fig. 3) are increased by $16 \mathrm{MW}$. This corresponds to 6, 2, and $8 \mathrm{MW}$ connected at busses 201, 205 , and 210, respectively. To demonstrate the benefits of the optimal storage sizing in combination with power factor control as well as other ANM schemes, a further $16 \mathrm{MW}$ of total controllable wind farms are connected to the network. They are located in a way that also exacerbates voltage issues (3, 10, and 3 MW at buses 201, 205, and 210, respectively). All the controllable generators are capable of operating with power factors between 0.95 inductive and capacitive according to UK requirements [20]. Table IV shows the size of each DG plant considered in this case study.

Similarly to section V-A, the Level 1 mono-period AC OPF is used to calculate the curtailment that would be needed if no other solution was available. The resulting curtailment is 113.8 MWh, i.e., $11.1 \%$ of the available resource for the studied week. The curtailment levels for the controllable DG at buses 201, 205 and 210 are 18.5\%, $11.1 \%$ and $3.8 \%$ respectively. To reduce curtailment, three potential sites for storage facilities are investigated. These sites correspond to the same locations of the controllable DG plants.

The optimal storage sizes found by the proposed approach are presented in Table $\mathrm{V}$ for $5 \%$ and $0 \%$ desired curtailment levels and considering different ANM schemes. A tolerance of $1 \%$ and a step of $1 \%$ are used. To quantify the benefit of adopting different ANM schemes on reducing the size of storage facilities, the overall capital cost is also presented in unit cost according to (1). The computing times required to find the optimal storage facilities are also presented.

For a 5\% desired curtailment and considering storage with unity power factor (Unity PF), no storage facility is placed at bus 210 due to the small volume of curtailment from the DG connected to this bus compared to the other sites. However, for $0 \%$ desired curtailment, storage facilities are required at all the potential locations to avoid curtailment. The storage at bus 205 has the largest power rating and energy capacity to solve the voltage issues at this bus in addition to congested lines 200-201 and 200-210. These two cases demonstrate the effective allocation of storage carried out by the approach but also the impact that a very strict curtailment can have $(0 \%$ curtailment is twice as expensive as with 5\%). 
TABLE V

Two Stage Storage Sizing (MVA/MWh)

\begin{tabular}{|c|c|c|c|c|c|c|c|c|c|c|c|c|c|}
\hline \multirow{3}{*}{$\begin{array}{c}\text { Storage PF } \\
\text { and } \\
\text { Curtailment }\end{array}$} & \multirow{3}{*}{$\begin{array}{l}\text { Additional } \\
\text { Flexibility }\end{array}$} & \multicolumn{6}{|c|}{$5 \%$ Desired Curtailment } & \multicolumn{6}{|c|}{ 0\% Desired Curtailment } \\
\hline & & \multicolumn{3}{|c|}{ Storage size per bus } & \multirow{2}{*}{$\begin{array}{c}\text { Total } \\
\text { MVA/MWh }\end{array}$} & \multirow{2}{*}{$\begin{array}{l}\text { Total } \\
\text { Unit } \\
\text { Cost }\end{array}$} & \multirow{2}{*}{$\begin{array}{l}\text { Time } \\
(\min )\end{array}$} & \multicolumn{3}{|c|}{ Storage size per bus } & \multirow{2}{*}{$\begin{array}{c}\text { Total } \\
\text { MVA/MWh }\end{array}$} & \multirow{2}{*}{$\begin{array}{l}\text { Total } \\
\text { Unit } \\
\text { Cost }\end{array}$} & \multirow{2}{*}{$\begin{array}{l}\text { Time } \\
(\min )\end{array}$} \\
\hline & & 201 & 205 & 210 & & & & 201 & 205 & 210 & & & \\
\hline Unity PF & - & $1.5 / 13.4$ & $3.3 / 15.2$ & - & $4.8 / 28.6$ & 31.8 & 45 & $1.9 / 20.5$ & $5.8 / 42.1$ & $0.6 / 1.7$ & $8.3 / 64.3$ & 69.9 & 16 \\
\hline $0.975 \mathrm{PFc}$ & - & $1.8 / 6.3$ & $3.1 / 13.5$ & - & $4.9 / 19.8$ & 23.1 & 52 & $2.0 / 24.6$ & $3.8 / 26.7$ & $1.1 / 4.8$ & $6.9 / 56.1$ & 60.7 & 19 \\
\hline $0.95 \mathrm{PFc}$ & - & $1.6 / 6.9$ & $3.4 / 9.4$ & - & $5.0 / 16.3$ & 19.6 & 51 & $2.3 / 25.9$ & $3.2 / 22.1$ & $1.3 / 5.8$ & $6.8 / 53.8$ & 58.4 & 21 \\
\hline Unity PF & OLTC & $1.5 / 6.8$ & - & - & $1.5 / 6.8$ & 7.6 & 49 & $2.8 / 30.6$ & - & $1.8 / 9.9$ & $4.6 / 40.5$ & 43.6 & 16 \\
\hline $0.975 \mathrm{PFc}$ & OLTC & $1.5 / 6.5$ & - & - & $1.5 / 6.5$ & 7.5 & 53 & $2.9 / 30.0$ & - & $1.9 / 9.8$ & $4.8 / 39.8$ & 43.0 & 18 \\
\hline $0.95 \mathrm{PFc}$ & OLTC & $1.5 / 6.4$ & - & - & $1.5 / 6.4$ & 7.4 & 58 & $2.9 / 29.9$ & - & $1.9 / 9.7$ & $4.8 / 39.6$ & 42.8 & 19 \\
\hline Unity PF & $\begin{array}{c}\text { OLTC + } \\
\text { DG PF }\end{array}$ & $1.1 / 4.9$ & - & - & $1.1 / 4.9$ & 5.6 & 58 & $2.8 / 28.5$ & - & $1.8 / 9.4$ & $4.6 / 37.9$ & 41.0 & 18 \\
\hline $0.975 \mathrm{PFc}$ & $\begin{array}{c}\text { OLTC + } \\
\text { DG PF }\end{array}$ & $1.1 / 4.8$ & - & - & $1.1 / 4.8$ & 5.5 & 59 & $2.8 / / 28.3$ & - & $1.8 / 9.4$ & $4.6 / 37.7$ & 40.8 & 18 \\
\hline $0.95 \mathrm{PFc}$ & $\begin{array}{c}\text { OLTC + } \\
\text { DG PF }\end{array}$ & $1.2 / 4.7$ & - & - & $1.2 / 4.7$ & 5.5 & 60 & $2.8 / 28.3$ & - & $1.8 / 9.4$ & $4.6 / 37.7$ & 40.8 & 18 \\
\hline
\end{tabular}

The use of the reactive power capabilities of storage facilities can also have an important role in managing voltages whilst reducing the energy capacity required. For 5\% desired curtailment, the total cost (as a proxy of the size) is reduced by $27 \%$ when controlling the power factor between 0.975 inductive/capacitive $(0.975 \mathrm{PFc})$. If the range is extended to 0.95 , the cost is reduced even further. However, for $0 \%$ desired curtailment, the benefits are smaller. This is because in this case congestion is a more pressing issue.

The extra flexibility provided by actively managing the OLTC at the BSP results in significantly smaller storage sizes. The overall capital cost of storage facilities for a desired curtailment of 5\%, is reduced by $76 \%$ when only using storage with unity power factor. Controlling the DG power factor, in addition to the OLTC, results in a much more flexible scheme able to achieve an even greater cost reduction of $82 \%$.

Furthermore, when using the OLTC, it can be seen that there is no storage facility placed at bus 205 as the voltage issues are solved mainly by better voltage targets at bus 200 .

Finally, it can be also noticed that the use of the reactive power capabilities of storage simultaneously with the OLTC and DG power factor does not provide further storage size reductions.

\section{A. Computational Intensity}

The computing time for all the cases in Section VI are presented in Table V. At 5\% desired curtailment level, all the cases require three iterations. This results in a computing time between 45 and 60 minutes. The most computationally intense cases are when more flexibility is considered. In contrast, around a third of the computing time is needed at $0 \%$ desired curtailment level since a single iteration is found to be enough to determine the sizes of storage facilities. For these cases, the computational intensity did not significantly increase with further flexibility. Finally, for a single iteration, the first stage (planning) requires around 3-5 minutes and the second stage (control) 12-20 minutes.

It is important to highlight that the computational intensity of the proposed approach is mostly dependent on the planning horizon and the corresponding number of samples. This can be seen in the case presented in Section V (single storage facility and single day planning horizon) where the computing time was significantly reduced to 7 minutes.

All the simulations presented in this and the previous sections here were carried out on a laptop with an Intel Core i7 $2.2 \mathrm{GHz}$ processor and $8 \mathrm{~GB}$ of RAM.

\section{INTEGRATED DG-STORAGE PlanNing}

In cases where planning studies are being carried out for the connection of new DG plants, the proposed planning framework can be adapted to find both the optimal capacities and locations of DG plants, and the sizes and locations of storage facilities. To do so, a new stage should be first introduced to evaluate the maximum DG capacity able to be connected to a distribution network considering different levels of energy curtailment and without using storage facilities. This preliminary stage is similar to the multi-period AC OPF presented in [2]. The obtained DG capacities can be then considered in the proposed two-stage planning framework to determine the optimal sizes of storage facilities required to reduce the curtailment previously considered. Ultimately, a decision has to be made based on the DG capacity, final curtailment level and the cost of storage.

\section{CONCLUSIONS}

This work presents a planning framework to find the minimum size of multiple storage facilities (power and energy capacities) to reduce wind power curtailment whilst managing congestion and voltages in distribution networks. The proposed two-stage iterative process incorporates near realtime control aspects to more accurately size storage facilities. The proposed approach is applied to a real UK $33 \mathrm{kV}$ network considering multiple wind farms as well as one-minute resolution wind and load profiles for a week.

The planning stage (first stage) was effective in producing initial storage sizes by adopting hourly profiles and a multiperiod AC Optimal Power Flow (OPF). From the control perspective (second stage), the results show the effectiveness of the proposed mono-period bi-level AC OPF in ensuring the 
adequate use of the charging, discharging, and idling modes of the storage facilities to minimize curtailment. This two-stage iterative process allowed the refinement of multiple storage sizes for a more accurate curtailment minimization.

The analysis also demonstrates the importance of high granularity data to truly quantify the curtailment level that could actually be achieved by a given storage facility. The use of simplistic hourly profiles was found to underestimate storage sizes (for the adopted wind and load profiles).

Furthermore, the benefits from incorporating further flexibility were also assessed. By using reactive power capabilities of storage facilities, it is possible to reduce storage sizes particularly when voltage issues prevail. The combined active management of OLTCs and power factor of DG plants resulted in the most significant benefits in reducing the required storage sizes.

To investigate the performance of different battery technologies, key parameters should be further investigated. This includes technical parameters, such as battery efficiency, maximum depth of discharge, etc. as well as non-technical, such as the power-energy cost relationship. Furthermore, the proposed planning framework can be extended to incorporate the effects of charging and discharging cycles on the lifetime of the storage facilities, either as constraints based on the battery characteristics or as a cost embedded in the objective function. In addition, further battery modelling aspects such as the non-linear relationship of voltages with the level of stored energy and, hence, the corresponding power limits for charging and discharging can also be incorporated.

\section{REFERENCES}

[1] R. A. F. Currie, G. W. Ault, C. E. T. Foote, and J. R. McDonald, "Active power-flow management utilising operating margins for the increased connection of distributed generation," IET Proceedings Generation, Transmission \& Distribution, vol. 1, no. 1, pp. 197-202, 2007.

[2] L. F. Ochoa, C. Dent, and G. P. Harrison, "Distribution network capacity assessment: variable DG and active networks," IEEE Transactions on Power Systems, vol. 25, no. 1, pp. 87-95, 2010.

[3] S. W. Alnaser and L. F. Ochoa, "Advanced network management systems: A risk-based AC OPF approach," IEEE Transactions on Power Systems, vol. 30, no. 1, pp. 409 - 418, 2015.

[4] J. P. Barton and D. G. Infield, "Energy storage and its use with intermittent renewable energy," IEEE Transactions on Energy Conversion, vol. 19, no. 2, pp. 441 - 448, 2004.

[5] N. Wade, P. Taylor, P. Lang, and J. Svensson, "Energy storage for power flow management and voltage control on an $11 \mathrm{kV}$ UK distribution network," in International Conference on Electricity Distribution (CIRED), 2009, pp. 1-4.

[6] J. A. Martinez, F. de Leon, A. Mehrizi-Sani, M. H. Nehrir, C. Wang, and V. Dinavahi, "Tools for analysis and design of distributed resources Part II: Tools for planning, analysis and design of distribution networks with distributed resources," IEEE Transactions on Power Delivery, vol. 26, no. 3, pp. 1653-1662, 2011.

[7] L. Alexio, G. Celli, E. Ghiani, J. Myrzik, L. F. Ochoa, and F. Pilo, "A general framework for active distribution network planning," in CIGRE Symposium 2013, pp. 1-8.

[8] Y. V. Makarov, P. Du, M. C. W. Kintner-Meyer, C. Jin, and H. F. Illian, "Sizing energy storage to accommodate high penetration of variable energy resources," IEEE Transactions on Sustainable Energy, vol. 3, no. 1, pp. 34-40, 2012.

[9] N. Etherden and M. H. J. Bollen, "Dimensioning of energy storage for increased integration of wind power," IEEE Transactions on Sustainable Energy, vol. 4, no. 3, pp. 546 - 553, 2013.

[10] S. Gill, I. Kockar, and G. W. Ault, "Dynamic optimal power flow for active distribution networks," IEEE Transactions on Power Systems, vol. 29, no. 1, pp. $121-1312014$.
[11] S. Carr, G. C. Premier, A. J. Guwy, R. M. Dinsdale, and J. Maddy, "Energy storage for active network management on electricity distribution networks with wind power," IET Renewable Power Generation, vol. 8, no. 3, pp. 249 - 259, 2014.

[12] Y. M. Atwa and E. F. El-Saadany, "Optimal allocation of ESS in distribution systems with a high penetration of wind energy," IEEE Transactions on Power Systems, vol. 25, no. 4, pp. 1815-1822, 2010.

[13] M. Nick, R. Cherkaoui, and M. Paolone, "Optimal allocation of dispersed energy storage systems in active distribution networks for energy balance and grid support," IEEE Transactions on Power Systems, vol. 29 , no. 5, 2014.

[14] G. Carpinelli, G. Celli, S. Mocci, F. Mottola, F. Pilo, and D. Proto, "Optimal integration of distributed energy storage devices in smart grids," IEEE Transactions on Smart Grid, vol. 4, no. 2, pp. 985 - 995, 2013.

[15] S. Schoenung, "Energy storage systems cost update," Sandia National Laboratories, Albuquerque, New Mexico, 2011.

[16] A. Gabash and P. Li, "Flexible optimal operation of battery storage systems for energy supply networks," IEEE Transactions on Power Systems, vol. 28, no. 3, pp. 2788 - 2797, 2013.

[17] Lithium-ion battery life. [Online]. Available: http://www.saftbatteries.com/battery-search/intensium\%C2\%AE-max

[18] J. Bisschop and M. Roelofs, "AIMMS - The User's Guide," Paragon Decision Technology, 2006.

[19] R. Dugan and T. E. McDermott, "An open source platform for collaborating on smart grid research," in IEEE/PES General Meeting, 2011, pp. 1 - 7.

[20] National Grid Electricity Transmission, The grid code 2012.

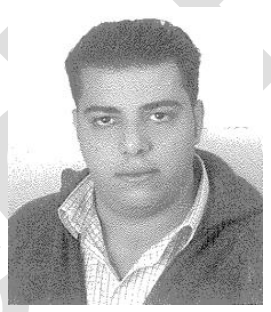

Sahban W. Alnaser (S'11) is a Research Associate in The University of Manchester, Manchester, U.K. $\mathrm{He}$ received the B.Sc. and M.Sc. degrees in Electrical Engineering from the University of Jordan, Jordan, in 2005 and 2008, respectively. He completed his Ph.D. studies from The University of Manchester, Manchester, U.K in 2015. His research interests include integration of distributed energy resources in distribution networks.

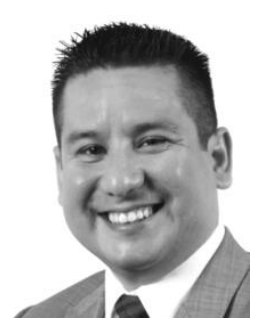

Luis F. Ochoa (S'01, M'07, SM'12) received the B.Eng. degree from UNI, Lima, Peru, in 2000 and the M.Sc. and Ph.D. degrees from UNESP, Ilha Solteira, Brazil, in 2003 and 2006, respectively. He is a Senior Lecturer in Smart Distribution Networks at The University of Manchester, UK. His current research interests include network integration of distributed energy resources and future low-carbon distribution networks. 